\title{
Estimativa da altura da camada limite planetária diurna e noturna na cidade de Natal, Estado do Rio Grande do Norte
}

\author{
Diagnostics of the diurnal and nocturnal planetary boundary layer height in Natal City, Rio Grande \\ do Norte State
}

\section{Roberta Everllyn Pereira Ribeiro', Maria Regina da Silva Aragão², Leandro Fontes de Sousa ${ }^{3}$, Magaly Correia de Fatima ${ }^{4}$ e Rafaella de Araújo Aires Vilar ${ }^{5}$}

\author{
${ }^{1}$ Pós-graduação em Meteorologia, CTRN/DCA, UFCG, Campina Grande, PB, Brasil \\ ${ }^{2}$ Profa Doutora efetiva, CTRN/DCA, UFCG, Campina Grande, PB, Brasil \\ ${ }^{3}$ Pós-graduação em Meteorologia, CTRN/DCA, UFCG, Campina Grande, PB, Brasil \\ ${ }^{4}$ Profa Doutora efetiva, CTRN/DCA, UFCG, Campina Grande, PB, Brasil \\ ${ }^{5}$ Pós-graduação em Meteorologia, CTRN/DCA, UFCG, Campina Grande, PB, Brasil \\ ${ }^{1}$ robertaeverllyn@hotmail.com
}

\begin{abstract}
Resumo
Este estudo tem como objetivo estimar a altura da camada limite planetária (CLP) e investigar características do seu desenvolvimento através de dados de sondagens atmosféricas realizadas às 00 UTC e 12 UTC em um mês chuvoso na cidade de Natal-RN. A altura da CLP é estimada aplicando os métodos dos perfis verticais de temperatura potencial e razão de mistura, para o período diurno e not urno. Foi possivel estimar a altura da CLP para quase todas as sondagens, na sua maioria utilizando os dois métodos. A altura média da CLP é menor no período noturno. A altura mais baixa (alta) estimada para o topo da CLP é $563 \mathrm{~m}$ (2847m). A altura da CLP é menor, em geral, na primeira quinzena do mês de estudo, maio de 2013.
\end{abstract}

Palavras-chave: CLP diurna, CLP noturna, temperatura potencial, razão de mistura, Estado do Rio Grande do Norte

\begin{abstract}
This study aims to estimate the PBL height and to investigate characteristics of its development by means of atmospheric sounding data obtained at 00 UTC and 12 UTC in a rainy month in the city of Natal-RN. The PBL height is estimated by applying the methods of the vertical profiles of potential temperature and mixing ratio, for daytime and nighttime. It was possible to estimate the PBL height for almost all soundings, by both methods in their majority. The mean PBL height is lower in nighttime. The lowest (highest) height estimated for the PBL top is $563 \mathrm{~m}$ (2847m). The PBL height is, in general, lower in the first half of the month of study, May 2013.
\end{abstract}

Keywords: diurnal PBL, nocturnal PBL, potential temperature, mixing ratio, Rio Grande do Norte State. 


\section{Introdução}

A Camada Limite Planetária (CLP), é a parte mais baixa da troposfera, diretamente influenciada pela superfície terrestre. Na CLP ocorrem as principais trocas de energia entre a superfície e a atmosfera controladas pelos transportes turbulentos (produção térmica e produção mecânica). O conhecimento sobre a variabilidade dessa camada, principalmente da sua altura, é fundamental para diversas aplicações como o controle e gerenciamento da qualidade do ar, estudos sobre mudanças climáticas, etc.

A altura da CLP não é constante, variando de dezenas de metros a poucos quilômetros, dependendo das modificações ocorridas na superfície terrestre, dos níveis de insolação diários, da hora do dia, da localização geográfica, dos fenômenos atmosféricos de grande e mesoescala, velocidade do vento, trocas de energia, e também pelos efeitos da interação da atmosfera com os diferentes tipos de superfície. À noite, com ventos fracos, a espessura da CLP é menor do que durante o dia, podendo atingir valores inferiores a $100 \mathrm{~m}$.

Este estudo tem como objetivo estimar a altura da CLP diurna e noturna através de dados de sondagens atmosféricas realizadas em um mês chuvoso na cidade de Natal, capital do Estado do Rio Grande do Norte, e investigar características do seu desenvolvimento.

\section{Metodologia}

Foram utilizados dados de sondagens atmosféricas de ar superior do mês de maio de 2013 realizadas às 00 UTC (21, hora local) e às 12 UTC (9, hora local) na estação de altitude de

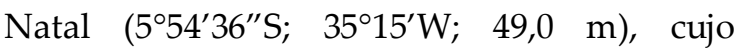
número sinótico é 82599 , e totais diários de precipitação observados na estação meteorológica de superfície do Instituto Nacional de Meteorologia (INMET) $\left(5^{\circ} 49^{\prime} 48^{\prime} \mathrm{S}\right.$, $\left.35^{\circ} 12^{\prime \prime} \mathrm{W}, 47 \mathrm{~m}\right)$. A coleta dos dados de precipitação é feita diariamente às 12 UTC pelo INMET.

A altura da CLP pode ser estimada por vários métodos cujos resultados podem diferir entre si, dependendo das condições atmosféricas reinantes. Neste estudo dois dos métodos de Seidel et al. (2010), descritos a seguir, foram utilizados. A altura da CLP é definida como a altura do nível no qual: (a) método (1) - o gradiente vertical da temperatura potencial é máximo, e (b) método (2) - o gradiente vertical da umidade especifica é mínimo. Naquelas situações em que o valor obtido pelos dois métodos difere é considerado o valor do método (1), tento em vista que erros associados com o sensor de temperatura são menos frequentes (SANTOS, 2005). Também há dois outros tipos de situação: (a) aqueles nos quais apenas um dos métodos possibilita estimar a altura, e (b) aquele no qual os dois métodos não indicam o topo da CLP. Neste trabalho foi utilizado o perfil da razão de mistura (r), já que é semelhante ao da umidade especifica (q) (VAREJÃO SILVA, 2006). Na estimativa da altura da CLP foi considerada a coluna atmosférica entre a superfície e o nível de $3000 \mathrm{~m}$. As sequências de sondagens que incluem aquela de CLP mais baixa e aquela para a qual não foi possível estimar a altura da CLP são discutidas.

\section{Resultados e Discussões}

No mês selecionado para estudo, maio de 2013, choveu um total de $253 \mathrm{~mm}$ na cidade de Natal, valor acima da média histórica (239,6 $\mathrm{mm})$ da cidade. Segundo o Boletim Climanálise (CPTEC/INPE), as chuvas que ocorreram no leste do Nordeste estiveram associadas principalmente aos episódios de Distúrbios Ondulatórios de Leste (DOL). Houve quatorze dias com registro de chuva em Natal, dos quais dois foram eventos de precipitação intensa, o primeiro na noite do dia 14 e o segundo na madrugada do dia 18, resultando num total pluviométrico diário de $85,1 \mathrm{~mm}$ (dia 15) e $96 \mathrm{~mm}$, respectivamente (Figura 1). 


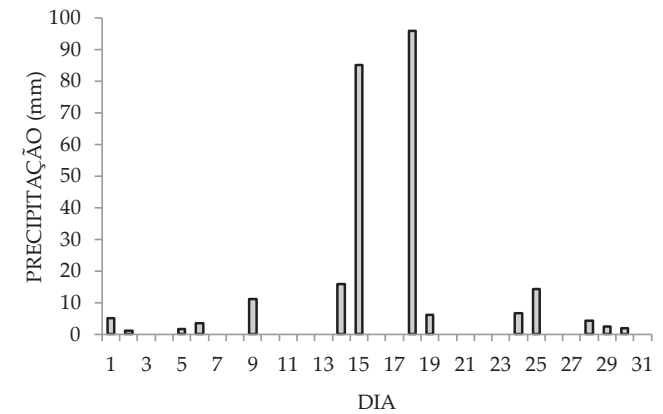

Figura 1 - Totais diários de precipitação (mm) observados em maio de 2013 na estação meteorológica de superfície do INMET localizada em Natal-RN. (Fonte dos dados: INMET-Instituto Nacional de Meteorologia).

$O$ vento em superfície obtido das sondagens variou entre os quadrantes sudoeste $(\mathrm{u}>0, \mathrm{v}>0)$ e sudeste $(\mathrm{u}<0, \mathrm{v}>0)$, com predominância do último (Figura 2). Os ventos mais intensos foram registrados nos dias $12(14,8 \mathrm{~km} / \mathrm{h})$ e $27 \quad(22,20 \mathrm{~km} / \mathrm{h})$ observados às 00 e $12 \mathrm{UTC}$, respectivamente. A menor velocidade do vento às 00 UTC foi registrada nos dias 15, 19, 25 e $30(3,7 \mathrm{~km} / \mathrm{h})$, e no dia $18(3,7 \mathrm{~km} / \mathrm{h})$, às 12 UTC. Em particular, os mínimos dos dias 15, 18 e 19 estão relacionados com os eventos de chuva forte na cidade

A altura estimada da CLP no mês de estudo teve no período noturno (00 UTC) uma média mensal mais baixa do que no período diurno (12 UTC) (Tabela 1). A maior altura estimada foi para as 12 UTC do dia $21(2847 \mathrm{~m})$, e a menor para as 00 UTC do dia $9(563 \mathrm{~m})$. Foi possível estimar a altura da CLP em quase todos os dias do mês analisado, a maioria através dos dois métodos propostos.

A sequência de sondagens que inclui a de CLP mais baixa é ilustrada na Figura 3. No dia 8 às 00 UTC o topo da CLP é baixo (579m). O perfil da temperatura potencial ilustra uma CLP quase neutra ( $\partial \theta / \partial z \sim 0)$ (Fig. 3a), acima da qual há uma camada estável $(\partial \theta / \partial z>0)$ com decréscimo acentuado na razão de mistura $(\partial r / \partial z<<0)(F i g .3 a, b)$. O vento é do quadrante sudeste com velocidade de 9,25 $\mathrm{km} \mathrm{h}^{-1} \mathrm{em}$ superfície (Fig. 2a). Às 12 UTC a altura da CLP é maior $(670 \mathrm{~m})$ devido ao aquecimento da superfície e fluxos turbulentos de calor no início da manhã (Fig. 3c), que resulta em uma camada com condição de instabilidade condicional $(\partial \theta / \partial z<0)$ próximo à superfície.
Acima dessa camada a condição é de quase neutralidade $(\partial \theta / \partial z \sim 0)$ até a altura de $670 \mathrm{~m}$. De maneira geral, a atmosfera é estável no restante do perfil. O perfil da razão de mistura (Fig. 3d) ilustra um forte gradiente vertical $(\partial \mathrm{r} / \partial \mathrm{z}<0)$ próximo à superfície. Acima dessa camada, entre $65 \mathrm{~m}$ e $170 \mathrm{~m}$, a razão de mistura aumenta. A umidade diminui nos níveis acima, até a altura de $1855 \mathrm{~m}$. O vento em superfície permanece do quadrante sudeste, com velocidade ligeiramente maior, de 11,10 $\mathrm{km} \mathrm{h}^{-1}$ (Fig. 2b).

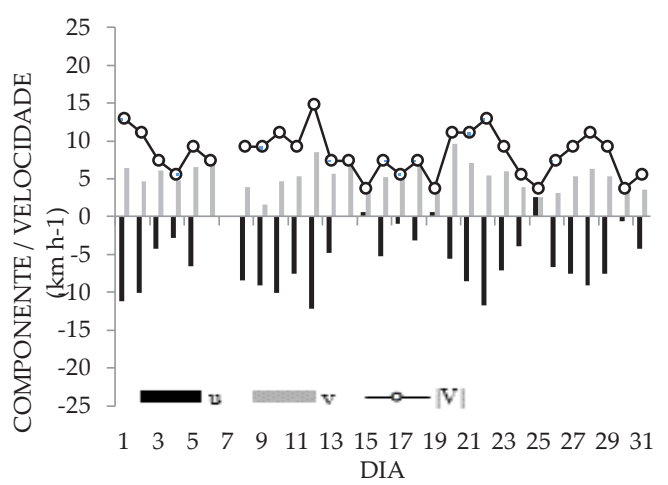

(a)

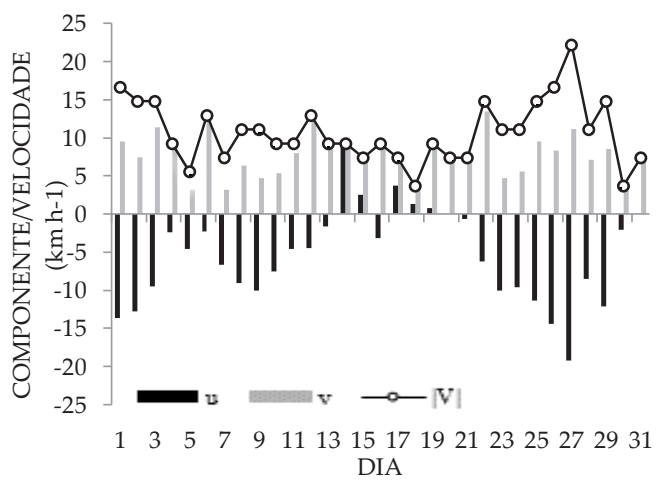

(b)

Figura 2 - Série temporal dos valores diários da velocidade $(|\mathrm{V}|)$ e das componentes zonal (u) e meridional (v) do vento à superfície $\left(\mathrm{km} \mathrm{h}^{-1}\right)$ em sondagens de ar superior realizadas na estação de altitude de Natal no mês de maio de 2013 RN às (a) 00 UTC e (b) 12 UTC. Os valores negativos (positivos) de $\mathrm{u}$ representam componente de leste (oeste). Os valores positivos de $\mathrm{v}$ representam componente de sul. Note a ausência do marcador no dia sem sondagem em (a). A simbologia utilizada é vista nos gráficos. (Fonte dos dados: www.weather.uwyo.edu/upperair/sounding.html). 
Tabela 1 - Altura estimada (m) da CLP às 00 e 12 UTC no mês de maio de 2013 na cidade de NatalRN. O traço assinala o horário sem sondagem e o asterisco, a sondagem para a qual não foi possível estimar a CLP com os métodos utilizados. Os valores em negrito e itálico são o mínimo e o máximo valor de altura (topo) da CLP. A hora local (HL) é HL= UTC - 3.

\begin{tabular}{|c|c|c|c|c|}
\hline \multirow{3}{*}{ DIA } & \multicolumn{4}{|c|}{ HORÁRIO } \\
\hline & \multicolumn{2}{|c|}{00 UTC } & \multicolumn{2}{|c|}{12 UTC } \\
\hline & ALTURA & MÉTODO & ALTURA & MÉTODO \\
\hline 1 & 895 & $(1)(2)$ & 1331 & $(1)(2)$ \\
\hline 2 & 978 & (1) (2) & 1070 & (1) (2) \\
\hline 3 & 1011 & (1) (2) & 1317 & (1) (2) \\
\hline 4 & 817 & (1) (2) & 1036 & $(1)$ \\
\hline 5 & 842 & (1) (2) & 940 & $(1)$ \\
\hline 6 & 1233 & (1) (2) & 1087 & (1) (2) \\
\hline 7 & - & - & 892 & (1) (2) \\
\hline 8 & 579 & (1) (2) & 670 & (1) (2) \\
\hline 9 & 563 & (1) (2) & 731 & (1) (2) \\
\hline 10 & 2039 & (1) (2) & 1026 & (1) (2) \\
\hline 11 & 1932 & (1) (2) & 1631 & (1) (2) \\
\hline 12 & 876 & (1) (2) & 1189 & (1) (2) \\
\hline 13 & 874 & (1) (2) & 621 & (1) (2) \\
\hline 14 & $*$ & $*$ & 1474 & (1) (2) \\
\hline 15 & 1527 & (1) (2) & 1678 & (1) (2) \\
\hline 16 & 2109 & (1) (2) & 1410 & (1) (2) \\
\hline 17 & 1631 & (1) (2) & 2215 & (1) (2) \\
\hline 18 & 1178 & $(1)$ & 1186 & $(1)$ \\
\hline 19 & 873 & (1) (2) & 2057 & (1) (2) \\
\hline 20 & 1540 & (1) (2) & 2016 & $(1)$ \\
\hline 21 & 2543 & (1) (2) & 2847 & (1) (2) \\
\hline 22 & 1517 & (1) (2) & 2299 & $(1)$ \\
\hline 23 & 573 & (1) (2) & 1533 & (1) (2) \\
\hline 24 & 1223 & (1) (2) & 2379 & (1) $(2)$ \\
\hline 25 & 2384 & (1) (2) & 2722 & $(1)$ \\
\hline 26 & 603 & (1) (2) & 670 & (1) (2) \\
\hline 27 & 807 & (1) (2) & 806 & (1) (2) \\
\hline 28 & 1922 & (1) (2) & 2040 & (1) (2) \\
\hline 29 & 2204 & (1) (2) & 1863 & (1) (2) \\
\hline 30 & 968 & (1) (2) & 1494 & (1) (2) \\
\hline 31 & 1294 & (1) (2) & 1855 & (1) (2) \\
\hline Média & 1294,3 & & 1486,6 & \\
\hline Desvio Padrão & 490,4 & & 504,3 & \\
\hline
\end{tabular}


No dia 9 às 00 UTC o topo da CLP é o mais baixo (563m) do mês analisado (Fig. 3e). Há uma camada estável rasa próxima à superfície, acima da qual a condição é de neutralidade até a altura de 563m, topo da CLP. Nessa camada a umidade diminui com o aumento da altura (Fig. 3f). O vento em superfície permanece do quadrante sudeste, com velocidade de 9,25 km $\mathrm{h}^{-1}$ (Fig. 2a). Às 12 UTC a altura da CLP é maior $(731 \mathrm{~m})$ e a atmosfera é instável $(\partial \theta / \partial z<0)$ na camada superficial (Fig. 3g) devido ao aquecimento na primeira metade da manhã. Nessa camada também há um forte gradiente vertical da razão de mistura $(\partial r / \partial z<<0)$, ilustrado na Figura 3 h, num padrão semelhante ao das 12 UTC do dia 8 (Fig. 3d). Acima do topo da CLP a atmosfera é estável na maior parte do perfil, porém há duas camadas instáveis rasas (Fig. 3g) nas quais a umidade cresce com o aumento da altura (Fig. 3h). O vento em superfície permanece do quadrante sudeste, com velocidade ligeiramente maior, de $11,10 \mathrm{~km} \mathrm{~h}^{-1}$ (Fig. 2b). Houve registro de precipitação neste dia (Fig. 1). A sequência de sondagens ilustrada na Figura 3 evidencia a estabilização da atmosfera no período noturno, na camada superficial, e a manutenção da CLP bem misturada. De maneira geral, o teor de umidade varia de $20 \mathrm{~g} \mathrm{~kg}^{-1}$ em superfície a um valor inferior a $10 \mathrm{~g} \mathrm{~kg}^{-1}$ na altura de $3000 \mathrm{~m}$.

A sequência de sondagens que inclui aquela em que não foi possível estimar a altura da CLP é ilustrada na Figura 4. No dia 13 às 00 UTC (Fig. 4a) a altura da CLP é de $874 \mathrm{~m}$, definida pelo topo de uma camada com condição de quase neutralidade e umidade quase constante $(\partial \theta / \partial z \sim 0, \partial r / \partial z \sim 0)$ (Fig. $4 a, b)$. Abaixo dessa camada, na camada superficial, a atmosfera é estável e a umidade decresce com o aumento da altura $(\partial \theta / \partial z>0, \partial r / \partial z<0)$. O vento em superfície é do quadrante sudeste, com velocidade de 7,40 $\mathrm{km} \mathrm{h}^{-1}$ (Fig. 2a). Às 12 UTC a altura da CLP, que é menor (621 m), é definida pelo topo de uma camada neutra com umidade constante $(\partial \theta / \partial z=0, \partial r / \partial z=0)$ (Fig. $4 c, d)$. Na camada superficial a atmosfera é instável e a umidade decresce com a altura $(\partial \theta / \partial z<0, \quad \partial r / \partial z<0), \quad u m$ padrão também encontrado nas sondagens diurnas ilustradas na Figura 3. O vento em superfície é do quadrante sudeste, com velocidade ligeiramente maior, de 9,25 $\mathrm{km} \mathrm{h}^{-1}$ (Fig. 2b).

No dia 14 às 00 UTC não foi possível estimar a altura da CLP pelos dois métodos propostos (Fig. 4e,f). Os perfis ilustram estabilidade e decréscimo da umidade com a altura, com variações verticais quase constantes cujas magnitudes diferem apenas na camada superficial, que apresenta gradientes mais fortes. O vento em superfície é de sul, com velocidade de 7,40 $\mathrm{km} \mathrm{h}^{-1}$ (Fig. 2a). Às 12 UTC a CLP é alta (1474 m) (Fig. 4g,h). O perfil da temperatura potencial ilustra uma camada estável na qual há diminuição da umidade com a altura, próximo à superfície (Fig. 4g,h). Acima dessa camada a atmosfera é neutra e a umidade é constante com a altura até o nível de $796 \mathrm{~m}$. Outra camada neutra é limitada pelos níveis de $1130 \mathrm{~m}$ e $1474 \mathrm{~m}$, topo da CLP (Fig. 4g). O vento em superfície é do quadrante sudoeste, com velocidade ligeiramente maior, de 9,25 $\mathrm{km} \mathrm{h}^{-1}$ (Fig. 2b). A estrutura vertical nas duas sondagens do dia 14 tem características bem distintas, com destaque para a ausência da camada espessa na qual a temperatura potencial e a razão de mistura são constantes e dos ventos de sudeste em superfície, vistos nas sondagens do dia 13. No dia 14 os ventos são de sul e sudoeste. $\mathrm{Na}$ noite desse dia ocorre o primeiro evento de chuva intensa do mês (Fig. 1). No caso das 12 UTC a evolução da CLP foi influenciada por transportes turbulentos associados com a variação do vento incluindo efeitos em superfície e circulações de mesoescala. 


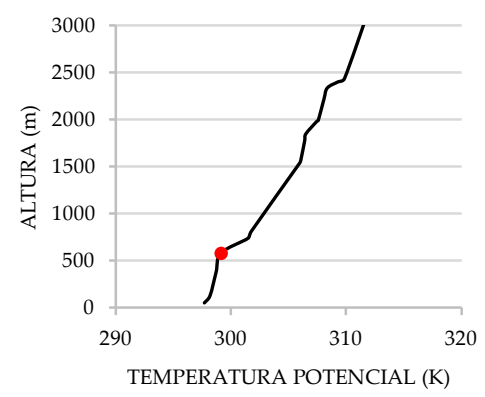

(a)

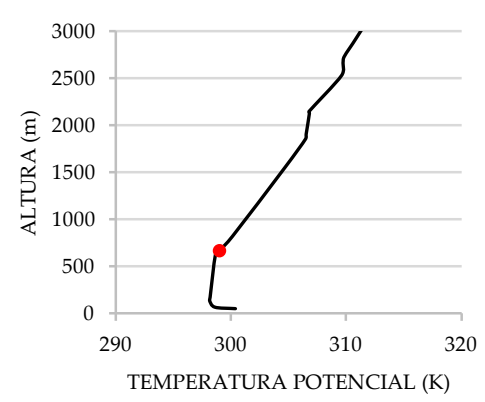

(c)

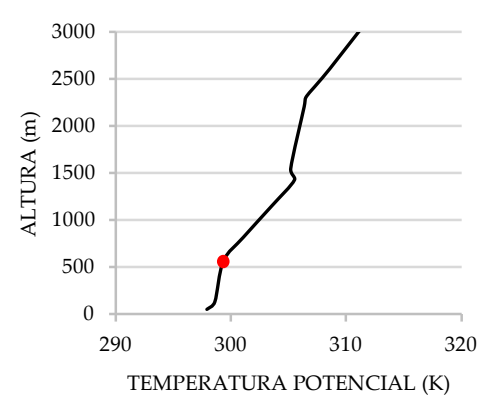

(e)

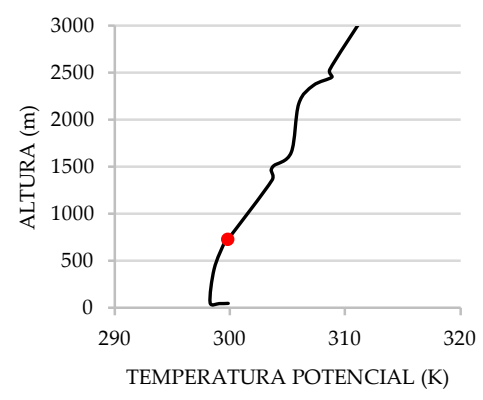

(g)

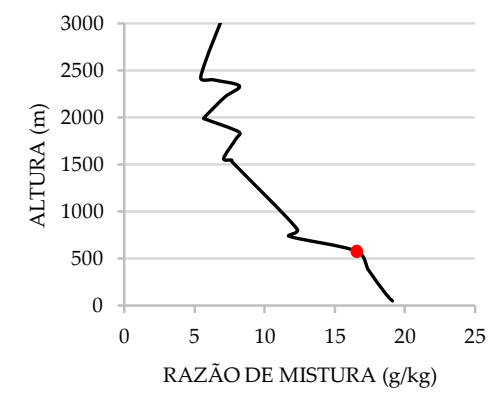

(b)

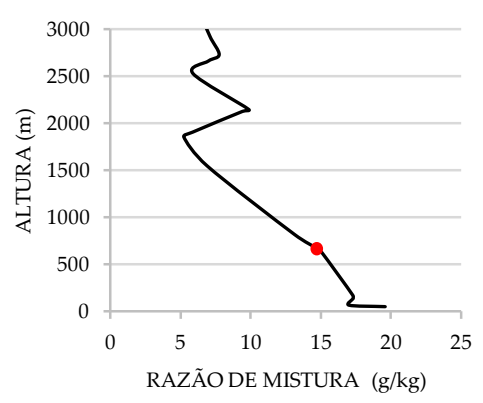

(d)

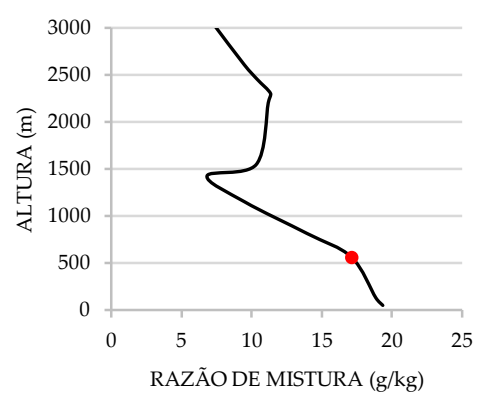

(f)

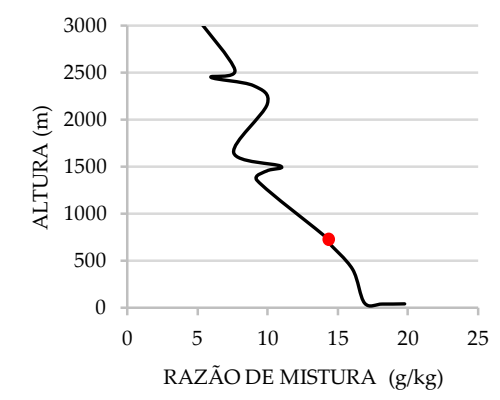

(h)

Figura 3 - Perfil vertical da temperatura potencial (à esquerda) e da razão de mistura (à direita) obtido de sondagem de ar superior realizada em maio de 2013 em Natal-RN, no dia: (a, b) 8 às 00 UTC, (c, d) 8 às 12 UTC, (e, f) 9 às 00 UTC e (g, h) 9 às 12 UTC. O círculo vermelho indica o topo da CLP. A altura zero nos perfis representa a superfície (49m). (Fonte dos dados: www.weather.uwyo.edu/upperair/sounding.html). 


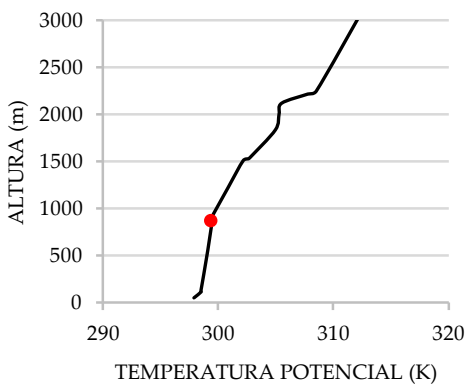

(a)

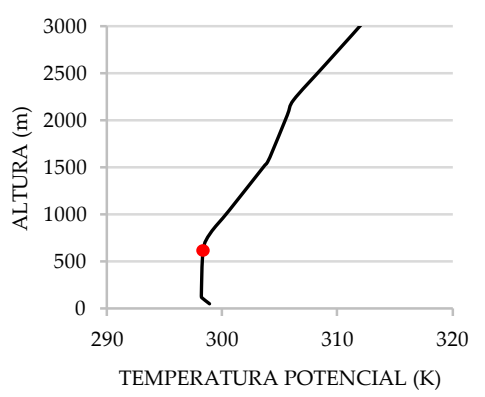

(c)

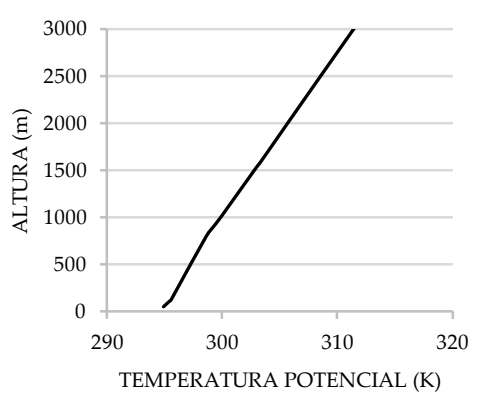

(e)

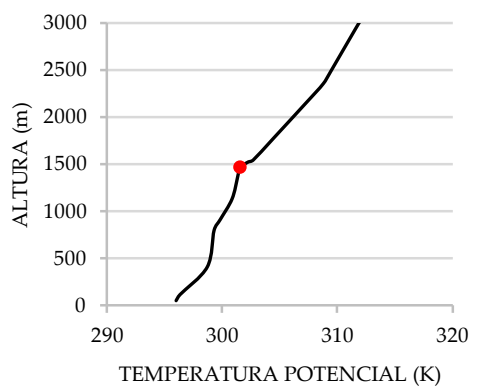

(g)

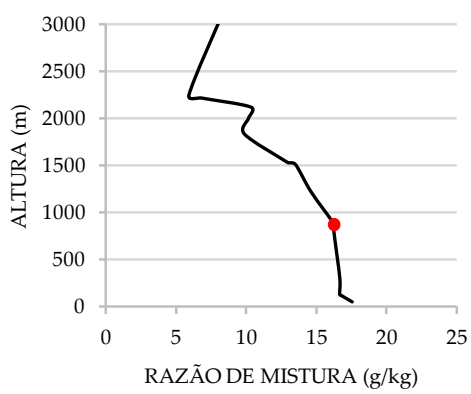

(b)

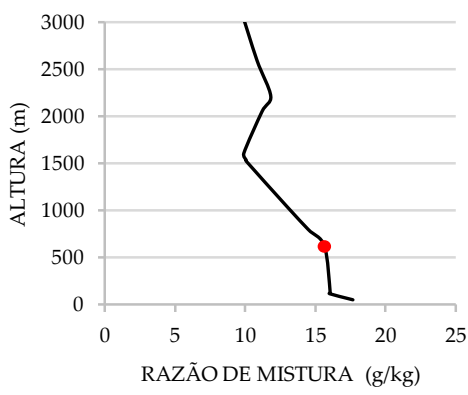

(d)

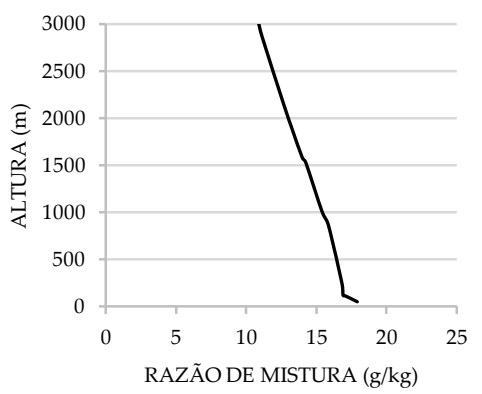

(f)

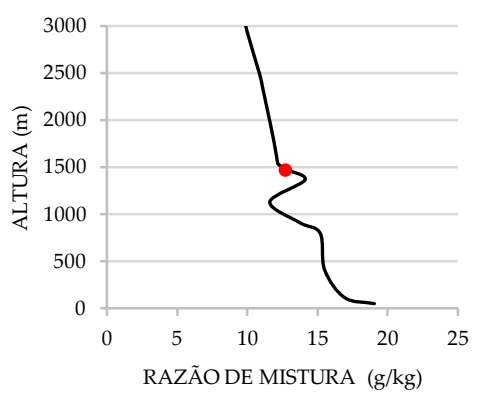

(h)

Figura 4 - Perfil vertical da temperatura potencial (à esquerda) e da razão de mistura (à direita) obtido de sondagem de ar superior realizada em maio de 2013 em Natal-RN, no dia: $(a, b) 13$ às 00 UTC, $(c, d) 13$ às 12 UTC, (e, f) 14 às 00 UTC e (g, h) 14 às 12 UTC. O círculo vermelho indica o topo da CLP. A altura zero nos perfis representa a superfície (49m). (Fonte dos dados: www. weather.uwyo.edu/upperair/sounding.html). 


\section{Conclusões}

A altura da CLP é estimada e características do seu desenvolvimento são analisadas usando sondagens atmosféricas diurnas e noturnas realizadas no mês chuvoso de maio de 2013, na cidade de Natal, capital do Estado do Rio Grande do Norte.

Foi possível estimar a altura da CLP para quase todos os dias do mês. A altura média da CLP no período noturno é menor. A altura mais alta (baixa) estimada é $2847 \mathrm{~m}$ (563m). A altura da CLP é menor, em geral, na primeira quinzena do mês de estudo.

\section{Agradecimentos}

A primeira autora agradece ao Conselho Nacional de Desenvolvimento Científico e Tecnológico (CNPq), e o terceiro e quinto autores à Coordenação de Aperfeiçoamento de Pessoal de Ensino Superior (Capes), pela concessão de bolsa de estudos.

\section{Referências}

CLIMANÁLISE - Boletim de Monitoramento e Análise Climática. Cachoeira Paulista, SP: INPE/CPTEC, v. 28, n. 05, mai 2013, Disponível em:

$<$ http://climanálise.cptec.inpe.br/ rclimanl/bole tim/pdf/pdf13/mai13.pdf $>$. Acesso em 13 jul. 2015.

SANTOS, L. A. R. Análise e caracterização da camada limite convectiva em área de pastagem, durante o período de transição entre a estação seca e chuvosa na Amazônia. (Experimento RACCI-LBA/Rondônia). 2005. 122 f. Dissertação (Mestrado em Meteorologia) - Instituto Nacional de Pesquisas Espaciais INPE, São José dos Campos.

SEIDEL, D. J.; AO, C. O.; LI, K. Estimating climatological planetary boundary layer heights from radiosonde observations: Comparison of methods and uncertainty analysis. Journal of Geophysical Research, 115, n. D16, p. D16113, 2010.
VAREJÃO SILVA, M. A. Meteorologia e Climatologia, versão digital 2, Recife-PE, p. 146-147, mar. 2006. 